\title{
Patterns of maternal and paternal inheritance in Russian populations
}

\author{
Anton Logachev \\ Dobzhansky Center for Genome \\ Bioinformatics \\ St. Petersburg State University \\ St. Petersburg, Russia \\ a.logachev@spbu.ru
}

\author{
Daisuke Hirata \\ Dobzhansky Center for Genome \\ Bioinformatics \\ St. Petersburg State University \\ St. Petersburg, Russia \\ d.khirata@spbu.ru
}

\author{
Gaik Tamazian \\ Dobzhansky Center for Genome \\ Bioinformatics \\ St. Petersburg State University \\ St. Petersburg, Russia \\ g.tamazian@spbu.ru
}

\begin{abstract}
Genome Russia (GR) study is a national collaboration to expand our understanding of demographic history of peoples of Russia, to get deep insight into population genetics and to create a detailed genetic map of a country. Here we report analyses of matrilineal and patrilineal inheritance derived from whole genome sequencing data collected in the framework of the project. We examined sequences of $171 \mathrm{Y}$ chromosomes and $322 \mathrm{mtDNA}$ ascertained from 14 ethnicities. We determined haplogroups for both Y-chromosomes and mtDNA sequences and estimated their distribution across the country. We performed phylogenetic and population genetic analyses for mtDNA and Y-chromosomes separately and then compared the results to reveal genetic history of indigenous groups living in Russia.
\end{abstract}

Keywords - Genome Russia Project, Y-chromosome, mtDNA, haplogroups

\section{Introduction}

To decipher the population and demographic history of indigenous Russian ethnicities, migratory routes, and settlings of humankind in Russia, we analyzed mitochondrial genomes and Y-chromosome sequences from WGS datasets generated in the course of GR study. Phylogenetic and population genetic analyses were performed to reveal the maternal and paternal genetic history of the indigenous ethnic groups in Russia. By comparing both mitochondrial and Ychromosomal DNA results, we inferred differences in the genetic history of both maternal and paternal population divergence and migration histories.

\section{Materials and methods}

We performed population genetic analyses for unrelated individuals (243 mtDNAs and $171 \mathrm{Y}$-chromosomes) from 14 populations (Central Russians, Southern Russians, Western Russians, Northern Russians, Adygeys, Seto, Komi, Udmurts, Khanty, Tatars, Bashkirs, Yakuts, Kalmyks, and Udege).

To assemble and genotype mitochondrial genomes we applied the MToolBox v.1.1 pipeline. In subsequent analyses we only used biallelic sites. To obtain mtDNA haplogroup information from each individual, the mtDNA haplogroup assignment of 322 individuals was searched by Haplogrep2 [2] using variant information generated with MToolBox as an input and PhyloTree.org - mtDNA tree Build $17[3,4]$ as a reference. To identify Y-chromosomal haplogroups in males we performed SNP calling, and then tested and used different publicly available software tools e.g., AMY-tree [5], YFitter [6], Yleaf [7], and assembled them into our own pipeline. We then used available results from previous studies and $1 \mathrm{~K}$ Genome project to construct phylogenetic trees for newly identified haplogroups in studied ethnicities.
To elucidate the population genetic status of each population, molecular diversity indices (Haplotype diversity, mean number of pairwise differences, number of segregating sites), Neutrality test (Tajima's $D$ ), and population pairwise $F_{S T}$ for the whole mtDNA sequences and for male-specific regions in Y-chromosome were calculated with Arlequin v.3.5.2.2 [8]. Multi-dimensional Scaling (MDS) based on population pairwise $F_{S T}$ genetic distances was conducted using 'cmdscale' function as implemented in R.

\section{Results}

We identified mtDNA haplogroups (Macro-haplogroup, Major haplogroup, and Haplogroup) distribution of the 243 individuals. Our analyses demonstrate that haplogroup composition of three populations, Yakuts, Kalmyks, and Udege was distinct from those of the remaining populations. Thus, macro-haplogroup M, which was mainly found in Asia and Siberia, is dominant in Yakuts, Kalmyks and Udege, while macro-haplogroup $\mathrm{R}$, which was mainly found in Europe, is dominant in the remaining populations examined in the study, including ethnic Russians. Interestingly, Udege can be identified by a unique major haplogroup combination (M7, M8, and N9), which is only found in this ethnicity among the 14 populations analysed.

Ethnic Russians, belonging to the East Slavs ethnic group are clustered together with Komi and Seto, the Finno-Ugric peoples, as revealed with the MDS plot. Surprisingly, according to our results, Tatars, being a Turkic nation, are closely related to their geographic neighbors, Khanty and Udmurts (the Finno-Ugric peoples), rather than to the members of the same Turkic language family Yakuts and Bashkirs. Adyghe, Kalmyk, and Udege peoples attributed to Circassians, Mongolic, and Tungusic language families, respectively, show similarity between genetic and linguistic distinctness. Overall, mtDNA from non-ethnic Russians is heterogeneous compared to the homogenous ethnic Russians. The Y-chromosome analyses shows that while subclades of R1a1 and R1b1 haplogroups are common for all ethnic Russian populations, the central and northern Russians also have a significant proportion of N1a haplogroup. Other Ychromosomal haplogroups in Russians are less-frequent. Komi and Khants both have a typical Finno-Ugric N1a haplogroup. Adyghe people have different subclade of G2a haplogroup commonly encountered in the Caucasus. Haplogroup frequency patterns revealed in Bashkirs show equal distribution of $\mathrm{J} 1, \mathrm{~J} 2 \mathrm{a}, \mathrm{E} 1 \mathrm{~b}$ and $\mathrm{C}$. We also noticed that Y-chromosomal haplogroups diversity is higher in populations of ethnic Russians and Bashkirs as compare to Adyghe and Komi people. 
In this study we examined recent genetic history of mtDNA and Y-chromosome haplotypes in the gene pool of 14 ethnicities from present day Russia and documented divergence across various populations.

\section{AKNOWLEDGMENTS}

We are grateful to all collaborators from Genome Russia Consortium for their valuable comments and input. We further acknowledge all the donors contributing to the project.

\section{REFERENCES}

[1] Calabrese, C. et al. MToolBox: A highly automated pipeline for heteroplasmy annotation and prioritization analysis of human mitochondrial variants in high-throughput sequencing. Bioinformatics (2014)
[2] Weissensteiner, H. et al. HaploGrep 2: mitochondrial haplogroup classification in the era of high-throughput sequencing. Nucleic Acids Res. (2016)

[3] van Oven, M. \& Kayser, M. Updated comprehensive phylogenetic tree of global human mitochondrial DNA variation. Hum. Mutat. 30, 386394 (2009).

[4] van Oven, M. PhyloTree Build 17: Growing the human mitochondrial DNA tree. Forensic Sci. Int. Genet. Suppl. Ser. (2015).

[5] Van Geystelen et al. AMY-tree: an algorithm to use whole genome SNP calling for $\mathrm{Y}$ chromosomal phylogenetic applications. BMC Genomics 14:101 (2013).

[6] Karafet et al. New binary polymorphisms reshape and increase resolution of the human Y chromosomal haplogroup tree. Genome Res. 18: 830-838 (2008).

[7] Ralf et al. Yleaf: Software for human Y-chromosomal haplogroup inference from Next-Generation Sequencing data. Mol. Biol. Evol. 35(5): 1291-1294 (2018).

[8] Excoffier, L. \& Lischer, H. E. L. Arlequin suite ver 3.5: A new series of programs to perform population genetics analyses under Linux and Windows. Mol. Ecol. Resour. 10, 564-567 (2010). 